\title{
Corticolous myxobiota of the Pernambuco Center of Endemism, Brazil
}

\author{
David Ítallo Barbosa $a^{12^{\star}}$, Andrea Carla Caldas Bezerra', Vitor Xavier de Lima ${ }^{1,2}$ and Laise de Holanda Cavalcanti,.2
}

Received: June 15, 2016

Accepted: September 23, 2016

\begin{abstract}
Situated within the Atlantic Forest domain, the Centro de Endemismo Pernambuco (Pernambuco Center of Endemism) - CEPE is regarded as a priority for biodiversity conservation worldwide. Covering an area of approximately 56,000 $\mathrm{km}^{2}$, it encompasses part of the states of Alagoas, Pernambuco, Paraíba and Rio Grande do Norte in northeastern Brazil. Here we present an identification key to 73 species of Myxomycetes that compose the known corticolous myxobiota of the Dense Ombrofilous Lowland Forest, Semideciduous Lowland Forest, Restinga Forest and Mangrove environments of CEPE. Specimens included here were found developed in natural conditions in the field or obtained through moist chamber culture. Among the species found, Arcyria cinerea, Clastoderma debaryanum, Cribraria confusa, C. violacea and Echinostelium minutum were the most common; and Comatricha longipila, Licea pedicellata, Paradiachaeopsis longipes, Perichaena calongei and Stemonaria fuscoides were the most rare. Our data indicates that the species richness of corticolous myxomycetes in rainforests is lower than that of temperate forests. These same data also suggest that taxonomic diversity (mean number of species per genus) is similar to, or higher than, that found in temperate regions of the world.
\end{abstract}

Keywords: Atlantic Forest, biodiversity, microhabitat, Myxogastria, Myxomycetes

\section{Introduction}

Myxomycetes (Amoebozoa) are unicellular phagotrophic eukaryotes that prey on fungus and bacteria, and occur in terrestrial environments as haploid, uninucleated myxamoebae and swarm cells or as diploid multinucleated plasmodium that produce fungus-like fruiting bodies (Gray \& Alexopoulos 1968). About one third of the species of Myxomycetes have worldwide distribution, but some seem to be restricted to the tropics and others to temperate regions (Stephenson et al. 2008). Studies of soil microbiota shows the importance of this group of organisms to the ecosystems where they occur, serving as regulators of microbial populations of bacteria, yeasts and filamentous fungi and in nutrient cycling and mineralization (Keller \& Everhart 2010; Stephenson \& Feest 2012).

Myxomycetes are classified into ecological groups according to the microhabitats where they develop and sporulate, being lignicolous and foliicolous myxomycetes the most well-known (Ing 1994). Corticolous myxomycetes inhabit the bark of living shrubs and trees. Species typical of this ecological group generally produce sporangia up to $1 \mathrm{~mm}$ in height and usually have protoplasmodia ( $\leq 0.5$ $\mathrm{mm}$ ), which have low mobility and are capable of responding

\footnotetext{
${ }^{1}$ Laboratório de Myxomycetes, Departamento de Botânica, Centro de Biociências, Universidade Federal de Pernambuco, 50.670-420 Recife, PE, Brazil

2 Programa de Pós-Graduação em Biologia de Fungos, Departamento de Micologia, Centro de Biociências, Universidade Federal de Pernambuco, 50.670-420 Recife, PE, Brazil

*Corresponding author: daviditallo-bio@hotmail.com
} 
rapidly to environmental conditions that induce sporulation (Everhart \& Keller 2008). Species of this group are not distributed randomly among the different environments where they are recorded, and neither occur with the same abundance throughout the year since their trophic stages are strongly influenced by temperature, moisture and substrate $\mathrm{pH}$ as well food availability (Madelin 1984; Tran et al. 2006). There is no evidence of endemism among species of myxomycetes, but differences in myxobiota composition between different types of microhabitats have already been observed (Novozhilov et al. 1999; 2003; 2006; Stephenson et al. 2000; Schnittler 2001).

Studies performed in tropical forests, which are characterized by high levels of pluviosity and air humidity, suggests that their myxomycete diversity is lower than that observed in temperate forests, and with low corticolous species richness (Stephenson et al. 2004). Analysing the composition of the corticolous myxobiota from temperate moist forest in the Great Smoky Mountains National Park (GSMNP), Snell \& Keller (2003) recorded 84 species from moist-chambers mounted with samples obtained from 25 trees. In urban environments of the province of Madrid, Spain, 37 species were documented from 81 moist-chamber cultures mounted with bark of living Quercus ilex (Fagaceae) (Basanta 1998).

Studies focusing on the corticolous myxobiota of Brazil were initiated by Cavalcanti (1974), who recorded 20 species on the bark of 300 trees of the Cerrado biome, of which 13 taxa were obtained through moist-chamber culture. Bezerra \& Cavalcanti (2007) listed ten species found on 20 specimens of Terminalia cattapa (Combretaceae), during 12 months of study in urban environments in Recife, Pernambuco. In Restinga of the Parque Estadual Dunas do Natal, Bezerra et al. (2007) obtained only 12 species from 200 moistchamber plates prepared with cortex of trees typical of this vegetation.

Distributed along the latitude of $28^{\circ}$, the Atlantic Forest is the second largest Neotropical forest, and is considered a biodiversity hotspot with numerous endemic species of different taxa distributed among centers of Brazilian endemism, including Centro de Endemismo Pernambuco (CEPE) (Tabarelli et al. 2006). Currently, information on corticolous myxomycetes occurring in the northeastern part of the Atlantic Forest and its associated ecosystems are scarce (Silva \& Cavalcanti 2010; Damasceno et al. 2011; Cavalcanti et al. 2014).

Aiming to expand up on the existing knowledge of the ecosystem distribution of myxomycetes in the Neotropics, this paper presents 73 corticolous species found in different forest environments of the CEPE and provides a key for their identification, based on records made between the years of 1970 and 2016.

\section{Materials and methods}

\section{Study area}

Situated in the Atlantic Forest domain, CEPE comprises $\sim 56,4000 \mathrm{~km}^{2}$ distributed from north of the São Francisco River, Alagoas, northward to Rio Grande do Norte ( $5^{\circ} 00^{\prime}$ to $10^{\circ} 30^{\prime} \mathrm{S} ; 34^{\circ} 50^{\prime}$ to $37^{\circ} 12^{\prime} \mathrm{W}$ ) (Fig. 1). A humid tropical climate (Köppen's As'), prevails throughout CEPE, with Autumn-Winter rains and precipitation varying from 1,500 to 2,000 mm a year (Coimbra-Filho \& Câmara 1996; Roda \& Pereira 2006; Rolim et al. 2007; Silva et al. 2011). This forest fragment differs from other Atlantic Forests fragments by the influences from the Amazon rainforest and Atlantic Forest of the south and southeastern of the Brazil it receives (Tabarelli \& Santos 2004).

CEPE is comprised of a variety of different vegetation types, including Ombrophilous and Seasonal Semideciduous Forests, ecological tension areas and pioneer formations, where about $8 \%$ of the species of higher plants of the Brazilian Atlantic Forest occur, plus Restinga and mangroves (Tabarelli et al. 2006). According to Uchôa Neto \& Tabarelli (2002), 1,200 species of trees and shrubs comprise the CEPE macroflora, with some of them being endemic, such as Manilkara dardanoi Ducke (Sapotaceae), Couepia impressa Prance and C. pernambucencis Prance (Chrysobalanaceae).

Species listed in the present work are from the following localities within CEPE:

Paraíba: 1. Biological Reserve (REBIO) Guaribas, Mamanguape, $06^{\circ} 40^{\prime} 40^{\prime \prime} S$ and $35^{\circ} 12^{\prime} 47^{\prime \prime}$ W. Seasonal Semideciduous Forest (lowland), 3,378 ha.

Pernambuco: 2. Charles Darwin Ecological Refuge, Igarassu, $07^{\circ} 48^{\prime} 55^{\prime \prime} \mathrm{S}$ and $34^{\circ} 57^{\prime} 16^{\prime \prime} \mathrm{W}$. Dense Ombrophilous Forest (lowland), 60 ha; 3. REBIO Usina São José, Igarassu, $07^{\circ} 50^{\prime} 00.46^{\prime \prime}$ and $34^{\circ} 54^{\prime} 30.05^{\prime \prime}$ W. Seasonal Semideciduous Forest (lowland), 323,3 ha; 4. REBIO Mata da Jaguarana, Paulista, $7^{\circ} 54^{\prime} 58.16^{\prime \prime}$ S and $34^{\circ} 51^{\prime} 24.83^{\prime \prime} W$. Seasonal Semideciduous Forest (lowland), 332, 8 ha; 5. REBIO Carnijó, Moreno, $8^{\circ} 06^{\prime} 50.96^{\prime \prime S}$ and $35^{\circ} 05^{\prime} 47.34^{\prime \prime W}$. Seasonal Semideciduous Forest (lowland), 135,5 ha; 6. Recife's Botanical Garden, Recife, 8 $04^{\prime} 42.15^{\prime \prime S}$ and 34 $57^{\prime} 28.34^{\prime \prime} \mathrm{W}$. Seasonal Semideciduous Forest (lowland), 27,0 ha; 7. Dois Irmãos State Park, Recife, 08 $00^{\circ} 33^{\prime \prime} S$ and $34^{\circ} 56^{\prime} 37^{\prime \prime} W$. Dense Ombrophilous Forest (lowland), 387,40 ha; 8. REBIO Gurjaú System forest, Cabo de Santo Agostinho, $8^{\circ} 14^{\prime} 21,7^{\prime \prime} S$ and $35^{\circ} 03^{\prime} 004^{\prime \prime} W$. Dense Ombrophilous Forest (lowland), 1.340,72 ha; 9. REBIO Saltinho, Rio Formoso and Tamandaré, $08^{\circ} 43^{\prime} 49.7^{\prime \prime} S$ and $035^{\circ} 10^{\prime} 54.0^{\prime \prime} \mathrm{W}$. Dense Ombrophilous Forest (lowland), 475 ha; 10. Rio Formoso Mangrove, Rio Formoso, $08^{\circ} 39^{\prime} 50^{\prime \prime} \mathrm{S}$ and $35^{\circ} 09^{\prime} 32^{\prime \prime} \mathrm{W}$. Mangrove, $12 \mathrm{Km}$.

Rio Grande do Norte: 11. Dunas do Natal State Park, Natal, $05^{\circ} 49^{\prime} 12^{\prime \prime} S$ and $35^{\circ} 11^{\prime} 16^{\prime \prime} W$, Restinga, 1,172 ha; 12. Private Reserve of Natural Heritage (RPPN) Mata Estrela, Baía Formosa, $06^{\circ} 22^{\prime} S$ and $35^{\circ} 00^{\prime} \mathrm{W}$. Restinga, 2, 365 ha. 


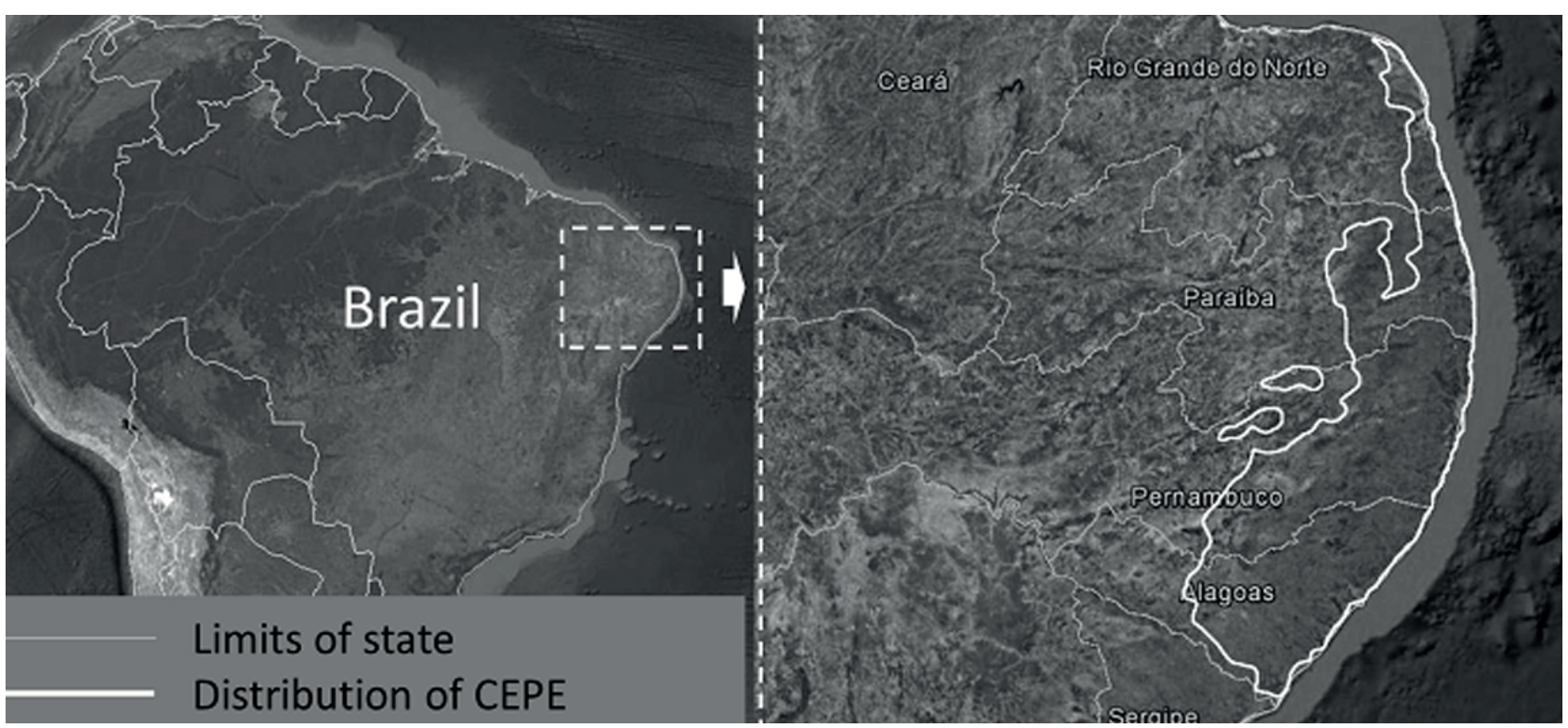

Figure 1. Localization of the Pernambuco Center of Endemism in the Northeastern Region of Brazil.

\section{Collection and identification}

Inventory was based on field collections, moist-chamber culture and specimens deposited in the herbaria UFP, URM and IPA, for which identifications were confirmed or redetermined, and binomials updated according to Lado (2015). The monographs of Martin \& Alexopoulos (1969), Farr (1976), Lado \& Pando (1997) and the keys and illustrations of Mitchell (2004) and Poulain et al. (2011) were used for species identification.

The taxonomic diversity index (S/G) was calculated according to Cavalcanti et al. (2014) and Stephenson et al. (1993).

\section{Results}

A total of 73 species was recorded from the cortex of living trees and shrubs of CEPE. The species belong to Myxogastromycetidae and Stemonitomycetidae and represent five orders, encompassing members of Clastodermataceae, Echinosteliaceae, Dictydiaethaliaceae, Reticulariaceae, Liceaceae, Arcyriaceae, Trichiaceae, Physaraceae, Didymiaceae and Stemonitaceae.

An identification key is presented for all the corticolous species recorded at CEPE. After the species and author names, numbers representing the localities where the species were recorded are given as superscripts.

\section{Identification key (index number show occurrence places at CEPE - see Study area]}

1. Lime absent, occasionally present on peridium surface 2

1'. Lime typically present in the sporophore as amorphous granules or crystals (Physarales)..................................... 17

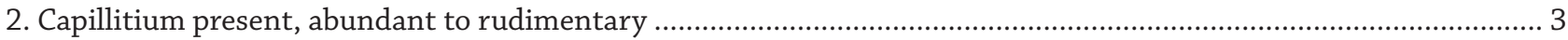

2'. Capillitium absent. Pseudocapillitium sometimes present (Liceales) .................................................................... 6

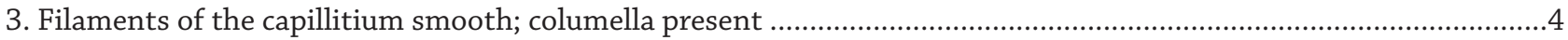

3'. Filaments of the capillitium ornamented with spirals, warts, spines, cogs, ridges, half-rings, rings and/or reticulations; columella absent (Trichiales)

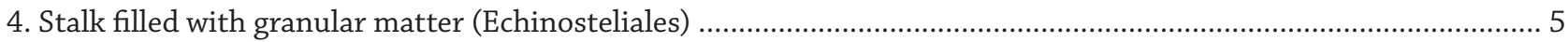

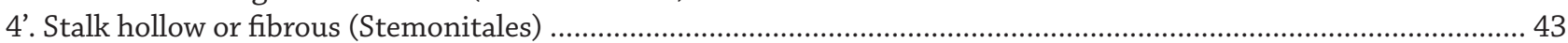

5. Sporocarp 1-1.2 mm total height; stalk brownish, filled with granular material bellow, translucent and tapering abruptly upwards after a droplet-like swelling; filament of the capillitium with membranous platelets attached at the tip; spores brown (Clastodermataceae).

Clastoderma debaryanum A. Blytt. ${ }^{(3,4,6,9,10,11)}$

5'. Sporocarp 0.25-0.55 mm total height; stalk hyaline, filled with granular material, tapering gradually upwards; filaments of the capillitium without platelets at the tip; spores hyaline (Echinosteliaceae) .... Echinostelium minutum de Bary. ${ }^{(1,3,4,5,5,6,9,12)}$ 6. Peridium persistent as a complete reticulum or a reticulum above and a calyculus bellow; dictydine granules always 
present in sporocarp (Cribrariaceae)

7. Peridial reticulum consisting of longitudinal and subparallel ribs, transversally connected by a slender filament; calyculus absent, rarely present Cribraria cancellata (Batsch) Nann.-Bremek. ${ }^{(4,7,12)}$

7'. Peridial reticulum consisting of a well-developed network 8

8. Sporocarp violaceous; calyculus deep, $1 / 2-2 / 3$ of sporotheca height

..Cribraria violacea Rex. ${ }^{(3,4,5,6,9,12)}$

8'. Sporocarp with brownish shades; calyculus up to $1 / 3$ of sporotheca height

9. Sporocarp yellowish-brown, up to $0.8 \mathrm{~mm}$ total height; nodes of peridial reticulum as small membranous junctions

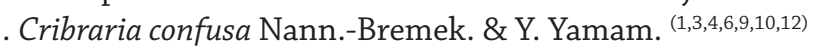
9'. Sporocarp reddish-brown, $1.5-3 \mathrm{~mm}$ total height; nodes of peridial reticulum well-defined

10. Stalk long, 6-12 times sporotheca diameter; peridial net without free ends, nodules thick, protruding, rounded ..... Cribraria microcarpa (Schrad.)Pers. ${ }^{(3,4,6,8,9,11,12)}$

10'. Stalk 3-5 times the sporotheca diameter; peridial net with (1) 2-4 (5) free ends, nodes angular

Cribraria intricata Schrad. ${ }^{(6,9,12)}$

11. Sporophore a pseudoaethalium; pseudocapillium present

11'.Sporophore sporocarpic or plasmodiocarpic; pseudocapillitium absent (Liceaceae) .............................................. 13

12. Mature pseudoaethalium with brownish shades, flat; dehiscence by hexagonal plates; pseudocapillitium filament connected at the extremities of peridial plates; spores pale yellow (Dictydiaethaliaceae)

Dictydiaethalium plumbeum (Schumach.) Rostaf. ${ }^{(2)}$

12'. Mature pseudoaethalium with greyish shades, subglobose; dehiscence by an apical pore; pseudocapillitium filament hyaline, free of the peridium; spores hyaline (Reticulariaceae) . Lycogala exiguum Morgan. ${ }^{(3)}$

13. Sporocarp sessile or plasmodiocarpic

13'. Sporocarp short or long stalked

14. Sporophore sporocarpic, sessile, wider than high; peridium dehiscence operculate, inner face smooth Licea kleistobolus G. W. Martin. ${ }^{(10,12)}$

14'. Sporophore plasmodiocarpic, elongate and fusiform, laterally compressed; dehiscence by a longitudinal preformed fissure; inner face of peridium ornamented . Licea biforis Morgan. ${ }^{(9)}$ 15. Spores in mass mostly dark; stalk, when present, short and nearly continuous with sporotheca; calyculus absent .. ..v Licea pedicellata (H.C. Gilbert) H.C. Gilbert. ${ }^{(9)}$

15'. Spores in mass mostly pale; stalk always present, sporotheca distinct from stalk; peridium persistent as a calyculus ... 16 16. Peridium single, dehiscence operculate, verrucose on the inner surface; spores hyaline, $8-12 \mu \mathrm{m}$ diam ..... Licea operculata (Wingate) G.W. Martin. ${ }^{(6,7,9)}$

16'. Peridium double, dehiscence by longitudinal plates, not verrucose on the inner surface; spores with pinkish shades 13.5-15 $\mu \mathrm{m}$ diam Licea erecta K.S. Thind \& Dhillon. ${ }^{(6,9)}$ 17. Lime granular or crystalline, present in sporophore (peridium, columella, stalk and/or hypothallus); capillitium not calcareous (Didymiaceae) ...

17'. Lime granular, present in sporophores; capillitium completely or partly calcareous (Physaraceae) .....

18. Stellate crystals (druses) present in peridium and sometimes columella; peridium typically single, less frequentely double

18'. Rhombic crystals or granular lime present in peridium, columella and/or stalk; peridium typically double, rarely single or triple 20 19. Sporotheca globose to hemispheric; stalk slender, about 2-3 times as long as sporotheca diam; peridium densely covered with white lime crystals; columella subglobose, brown, calcareous Didymium nigripes (Link) Fr. ${ }^{(4)}$ 19'. Sporotheca discoid; stalk rather thick, sometimes very short; peridium more or less covered with white lime crystals above, thickened and limeless on the underside; columella represented by thickened, discoid, sporotheca base .....

Didymium clavus (Alb. et Schwain.) Raben. ${ }^{(10)}$ 20. Sporophore plasmodiocarpic, flattened and lobate or sporocarp discoid, sessile to short-stalked, gray to grayishwhite; peridium with lime granules forming a thin crust; columella flesh-colored, sometimes rudimentary .....

Diderma chondrioderma (de Bary \& Rostaf.) G. Lister. ${ }^{(3)}$

20'. Sporophores sporocarpic, cylindric to ellipsoid, stalked, bluish or bronze; stalk and columella white, strongly impregnated with lime; peridium membranous, without lime, iridescent . Diachea leucopodia (Bull.) Rostaf. ${ }^{(10)}$ 
21. Sporophore a pulvinate aethalium, with a calcareous, well developed yellow cortex, usually solitary; pseudocapillitium present. Fuligo septica (L.) Wigg. ${ }^{(7)}$

21'. Sporophore a sporocarp or plasmodiocarp, isolated or gregarious; pseudocapillitium absent ............................. 22 22. Capillitium with tubular infoldings at peridium inner face, simple or forked, totally filled with lime granules, usually bifurcate, tapered to tips, filaments hyaline, short, without lime, generally forked and which attaches to the base ......... Badhamiopsis ainoae (Yamash.) T.E. Brooks \& H.W. Keller. ${ }^{(3)}$ 22'. Capillitium with rounded, fusiform or angular calcareous nodes, connected by hyalline filaments, without lime, short or long, simple or branched .

23. Dehiscence by preformed operculum or irregularly circumscissile; basal portion persisting as a calyculus (Craterium)

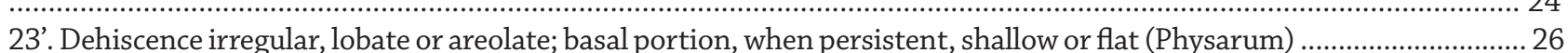

24. Sporocarp reddish-violet, fading to grayish-lilac; sporotheca globet-shaped or long and cylindric; pseudocolumella pale violet; capillitium pale violet, with large violet lime-knots .................................. Craterium paraguayense (Speg.) G. Lister. ${ }^{(7)}$ 24'. Sporocarp red-brown, golden-yellow or greenish; sporotheca globose, ovoid, obovoid, cylindric or turbinate; pseudocollumela yellowish or white; capillitium with white, yellow or ochraceous lime-knots .................................. 25 25. Sporotheca golden-yellow or greenish, fading almost to white, obovoid, ovoid or globose; peridium single, cartilaginous base with yellow lime-granules; pseudocollumela absent or represented by a yellow central mass; capillitium yellow, lime-knots yellow ..... Craterium aureum (Schum.) Rostaf. ${ }^{(7)}$ 25'. Sporotheca white or whitish above and with red-brown or ochraceous base, ovoid, cylindric or turbinate; peridium double, membranous; pseudocollumela calcareous, elongate, white or yellowish; capillitium yellow, lime-knots white, yellowish or ochraceous ............................................................................. Craterium leucocephalum (Pers.) Ditmar. ${ }^{(7)}$ 26. Sporophore sporocarpic, stalk not calcareous; peridium and capillitial nodes scarlet-red or pinkish; spores pinkishbrown Physarum roseum Berk. \& Broome. ${ }^{(3,11)}$ 26'. Sporophore sporocarpic or plasmodiocarpic, white or with grayish, brownish, yellowish or orange shades; spores brown

27.

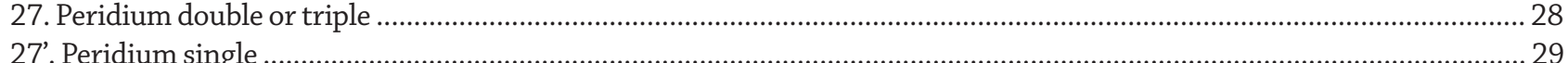

28. Plasmodiocarp strongly laterally compressed; peridium chalky white, double, outer layer smooth, calcareous, inner layer membranous, iridescent; dehiscence apical by preformed fissure; spores with conspicuous spines

.. Physarum echinosporum Lister. ${ }^{9,11}$

28'. Plasmodiocarp not compressed; peridium brown to beige, triple, outer layer smooth, middle layer white, inner layer membranous, rugose, white to brownish; peridial layers breaking up in angular fragments above and triangular, reflexed lobes, laterally; spores nearly smooth to verruculose..... . Physarum bogoriense Racib. ${ }^{(7)}$

29. Pseudocolumella presente . 30

29'. Pseudocolumella absent 31

30. Stalk calcareous; dehiscence floriform; pseudocollumela calcareous, white, globose; capillitial nodes white, calcareous, small, fusiform to ovoid, connected by abundant hyaline filaments; spores 8-10 $\mu \mathrm{m}$ diam

Physarum stellatum (Massee) G. W. Martin. ${ }^{(6,7)}$

30'. Stalk not calcareous; capillitial nodes calcareous, small, rounded, densely aggregated in the center; spores 6.5-7.5 $\mu \mathrm{m}$ diam Physarum nucleatum Rex. ${ }^{(4,6)}$

31. Sporophore plasmodiocarpic or sporocarpic, sessile or short-stalked …………....................................................... 32

31'. Sporophore sporocarpic, stalk well-developed ........................................................................................................ 34

32. Plasmodiocarp in rosete-like mass, sometimes densely aggregated, occasionally mixed with contorted sporocarps ..

32'. Plasmodiocarp short, sometimes ramificated or ring-shaped to sporocarpic, sessile or short-stalked . Physarum gyrosum Rostaf. ${ }^{(8,9)}$

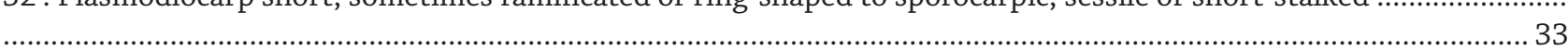

33. Sporophore orange to greenish-yellow, plasmodiocarpic, cylindric, sometimes ramificated or ring-shaped, or sporocarpic, sessile, pulvinate to subglobose, rarely short-stalked; peridium membranous, dotted with prominent glossy lime scales, nearly limeless basally; spores dark brown, verruculose. .................................................. Physarum auriscalpium Cooke. ${ }^{(10)}$ 33'. Sporophore yellow, plasmodiocarpic or sporocarpic, depressed-globose to pulvinate, sessile or short-stalked; calcareous yellow scales included in the rugose or areolate peridium; spores violaceous-brown, spinulose..... Physarum decipiens M. A. Curtis. ${ }^{(11)}$

34. Sporotheca with yellowish, greenish, orange or ochraceous shades ....................................................................... 35

34'. Sporotheca white or with grayish shades, rarely grayish-brown ................................................................... 37

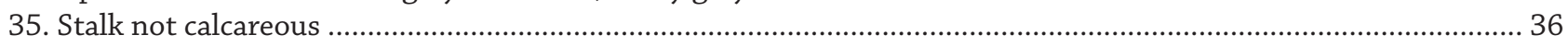


35'. Stalk calcareous

36. Sporotheca subglobose to lenticular, yellow, greenish to reddish-orange; peridium membranous, delicate, dehiscence areolate above, floriform below .....

Physarum viride (Bull.) Pers. ${ }^{(9,11)}$

36'. Sporotheca globose to depressed-globose or obovoid, yellow to ochraceous with brownish base; peridium thickened and persistent as a cup below ... Physarum oblatum T. Macbr. ${ }^{(7)}$ 37. Stalk white, rarely ochraceous, sometimes reddish below, up to half total height; dehiscence irregular; columella short, conic, white or yellowish, rarely orange..... Physarum melleum (Berk. \& Broome) Massee. ${ }^{(3)}$ 37'. Stalk pale yellow above, often becoming darker below, more than half of total height; dehiscence commonly by petaloid lobes; columella absent

Physarum tenerum Rex. ${ }^{(10)}$ 38. Sporocarp strongly compressed, reniform, fan-shaped or lobate .......................... Physarum compressum Alb. \& Schwein. ${ }^{(3,4)}$ 38'. Sporocarp globose, subglobose, depressed-globose, obovoid, cylindric, clavate, discoid, saucer-shaped or lenticular .... 39 39. Sporotheca globose, subglobose, obovoid, cylindric or clavate; dehiscence irregular 40 39'. Sporotheca depressed-globose to lenticular; dehiscence areolate, sometimes floriform ...................................... 42 40. Stalk calcareous, stout, tapering upward, white, rarely yellowish, up to half total height; peridium densely frosted with lime granules Physarum leucopus Link. ${ }^{(7)}$ $40^{\prime}$. Stalk not calcareous, fuscous to reddish-brown or black, $1 / 3$ to $2 / 3$ of total height; peridium flecked with lime squamules or marked with calcareous veins and dots, sometimes rather strongly calcareous .. 41 41.Columella cylindric, attaining the apex of the sporotheca, or short, clavate to conic, dark brown; capillitial nodes angular

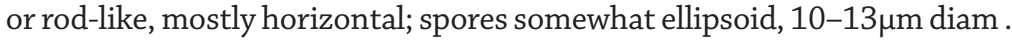
Physarum crateriforme Petch. ${ }^{(7)}$ 41'. Columella absent; capilitial nodes mostly rounded, or angular and branching; spores globose, $9-11 \mu \mathrm{m}$ diam ............. .Physarum leucopheum Fries. ${ }^{(7)}$ 42. Sporotheca depressed globose to lenticular; dehiscence areolate, annular or petaloid; stalk black at the base, tapering toward the white apex; spores (7) 8-9 (10) $\mu \mathrm{m}$ diam .................................................... Physarum album (Bull.) Chevall. ${ }^{(9)}$ 42'. Sporotheca discoid to saucer-shaped; stalk slender, twisted, translucent, reddish brown; peridium breaking areolately above; spores 10-17 $\mu$ m diam. Physarum pezizoideum (Jungh.) Pavill. \& Lagarde. ${ }^{(7)}$

43. Columella and stalk hollow, occasionally opaque and fibrous at base ................................................................... 44



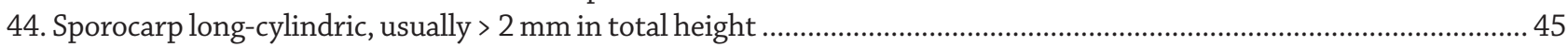

44'. Sporocarp globose to subglobose, usually < $2 \mathrm{~mm}$ in total height .................................................................... 52

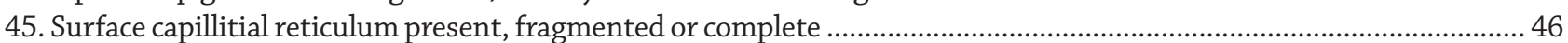

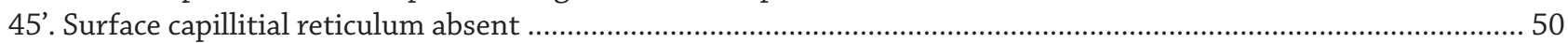

46. Peridium silvery, partially evanescent, persistent as patches and/or a basal cup; surface reticulum fragmented; spores typically with scattered, more or less prominent clusters of dark warts

Stemonitopsis typhina (F.H. Wigg.) Nann.-Bremek. ${ }^{(8)}$

46'. Peridium totally evanescent; surface reticulum complete; spores without clusters of prominent warts .......................... 47 47. Sporocarp fuscous to deep reddish-brown; spores verrucose-reticulate or spinulose-reticulate

Stemonitis fusca Roth. ${ }^{(3,4,10,11)}$

47'. Sporocarp dull cinnamon, rusty or purplish brown to nearly black; spores verrucose to nearly smooth ......................... 48 48. Surface reticulum with meshes mostly over $20 \mu \mathrm{m}$ in diameter; sporotheca dark purplish-brown to nearly black; columella becoming coiled and tortuous near apex ..... Stemonitis splendens Rostaf. ${ }^{(8)}$ 48'. Surface reticulum with meshes mostly up to $20 \mu \mathrm{m}$ in diameter; sporotheca rusty brown to dull cinnamon; columella becoming dissipated bellow apex

49. Sporocarps densely clustered; stalk ca. half of its total height; surface reticulum persistent; spores nearly smooth, 5-7.5 $\mu \mathrm{m}$ diam . Stemonitis axifera (Bull.)T. Macbr. ${ }^{(6)}$ 49'. Sporocarps in small clusters; stalk less than one third of its total height; surface reticulum persistent above; spores verrucose, 7-9 $\mu \mathrm{m}$ diam. Stemonitis herbatica Peck. ${ }^{(7)}$ 50. Columella flexuous, attaining apex of the sporotheca; capillitium without expansions and with numerous free ends .....

50'. Columella dissipating in 2-3 branches bellow apex; internal capillitium with many expansions, becoming thinner towards periphery, with rare free ends; spores brown, verrucose-reticulate

Stemonaria fuscoides Nann.-Brem. \& Y. Yamam. ${ }^{(10)}$

51. Sporangium up to $10 \mathrm{~mm}$ long, semi-erect; columella attaining apex where it may expand; capillitum flexuous, not dichotomously furcate, with hyaline free ends at the periphery; spores densely verrucose, often paler on one side ............... Stemonaria irregularis (Rex) Nann.-Bremek., R. Sharma\& Y. Yamam. ${ }^{(10)}$

51'. Sporocarp 10-50 mm long, pendent; capillitium rigid, horizontal, dichotomously branching; spores verrucose-reticulate 
52. Peridium evanescent, not iridescente

52'. Peridium persistent, iridescente

53. Capillitium lax, consisting of 3-4 main filaments, dichotomously branching, arising from apex of columella; spores with clusters of prominent dark warts, $6.5-8 \mu \mathrm{m}$ diam ..... Macbrideola martinii (Alexop. \&Beneke) Alexop. ${ }^{(9)}$

53'. Capillitium scarce, rudimentary; spores with warts varying in size and distribution, 7-9 $\mu \mathrm{m}$ diam Macbrideola decapillata H.C. Gilbert. ${ }^{(4,11)}$

54. Sporocarp up to $0.4 \mathrm{~mm}$ total height; capillitium absent to scarce; spores with evenly distributed warts. Macbrideola scintillans H. C. Gilbert. ${ }^{(9,10,11)}$

54'. Sporocarp > $0.4 \mathrm{~mm}$ total hight; capillitium abundant; spores with clusters of prominent warts Collaria arcyrionema (Rostaf.) Nann.-Bremek. ex Lado. (6,10,11)

55. Sporocarp usually $<2 \mathrm{~mm}$ total height; capillitia with filaments dichotomously branched, never or rarely anastomosed

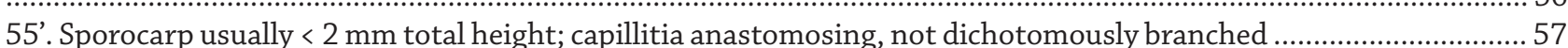
56. Stalk $8 / 10$ or more of total height; capillitium flexuous; spores $7-8 \mu \mathrm{m}$ diam

.Paradiacheopsis longipes Hooff \& Nann.-Bremek. ${ }^{(11)}$

56 . Stalk up to $2 / 3$ of total height; capillitium not flexuous; spores $8-10 \mu \mathrm{m}$ diam .....

57. Sporotheca fusiform; spores spinulose, with clusters of prominent spines

Paradiacheopsis rigida (Brândza) Nann.-Bremek. ${ }^{(1,9)}$ .. Comatricha tenerrima (M.A. Curtis) G. Lister. ${ }^{(4)}$

57'. Sporotheca globose to short-cylindric; spores verrucose, when spinulose, spines not clustered .............................. 58 58. Columela reaching at most the middle of the sporotheca; capillitium arising from the top of columela ........................ Comatricha elegans (Racib.) G. Lister. ${ }^{(7,11)}$

58'. Columella surpassing half of sporotheca; capillitium arising from the whole columella .............................................. 59

59. Capillitium anastomosed at periphery, without free ends...................................... Comatricha pulchella (C. Bab.) Rostaf. ${ }^{(10)}$

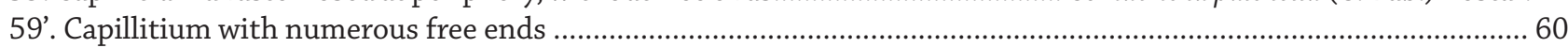

60. Capillitium with dichotomously branched filaments, rarely anastomosed; spores 6-7.5 $\mu \mathrm{m}$ diam ....

Comatricha longipila Nann.-Bremek. ${ }^{(11)}$

60'. Capillitium with filaments not dichotomously branched, anastomosed, forming internal loose reticulum; spores 8-11 $\mu \mathrm{m}$ diam Comatricha laxa Rostaf. ${ }^{(3,7)}$

61. Capillitium filaments always decorated with well-defined spirals (Trichiaceae) . .62

61 '. Capillitium filaments decorated with warts, spines, cogs, ridges, half-rings, rings and/or reticulations; spirals absent. (Arcyriaceae)..... 65

62. Sporophore plasmodiocarpic, branched, reticulate or ring-shaped; spores banded reticulate, bands non-pitted, border thickness $1-2 \mu \mathrm{m}$ . Hemitrichia serpula (Scop.) Rostaf. ${ }^{(7,8)}$

62'. Sporophore sporocarpic, stalked; spores spinulose to verrucose ......

63. Peridium single, membranous, remaining as a deep calyculus; inner face densely papilate or faintly subreticulated; stalk long, filled with cysts at base; capillitium filaments uniform in diameter; spores verrucose to spinulose, 6.5-7.5 (-8) $\mu \mathrm{m}$ diam ....... Hemitrichia calyculata (Speg.) M.L. Farr. ${ }^{(4,6,10,11)}$ 63'. Peridium double, inner layer attached to the outer layer, persistent but not forming a calyculus; inner surface papillate or faintly wrinkled; stalk short, rarely absent; capillitium filaments with expansions and constrictions ...................... 64 64. External layer of peridium gelatinous, with dark brown protuberances of refuse matter, persistent as brown pustules, inner layer membranous, papillate; spores $9-11 \mu \mathrm{m}$ diam

Hemitrichia pardina (Minakata) Ing. ${ }^{(4,9)}$ 64'. External layer of the peridium membranous, tightly adhered to membranous inner layer; spores $11-13 \mu \mathrm{m}$ diam ...

Hemitrichia minor G. Lister. ${ }^{(7)}$

65. Sporophore sporocarpic, with grayish or reddish shades; stalk filled with cysts; peridium single, persistent, the lower portion remaining as a calyculus

65 '. Sporophore plasmodiocarpic or sporocarpic, with brownish shades; stalk, if present, not filled with cysts; peridium

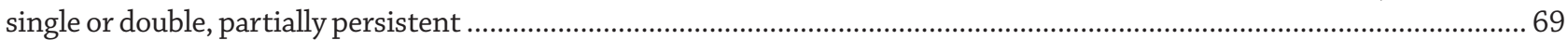
66. Capillitium remaining attached to the calyculus at maturity; sporotheca with reddish, grayish or yellowish shades; inner surface of calyculus decorated with warts, spines, ridges or fine reticulations, sometimes almost smooth ........................67 66'. Capillitium detached from calyculus at maturity; sporotheca reddish-brown to reddish-orange; inner surface of calyculus reticulate or alveolate. Arcyria ferruginea Sauter. ${ }^{(7)}$ 67. Sporotheca with reddish shades; capillitium decorated with warts or spines, cogs, ridges, rings or half-rings, helicoidally arranged . Arcyria denudata (L.) Wettst. ${ }^{(9)}$ 
David Ítallo Barbosa, Andrea Carla Caldas Bezerra,

Vitor Xavier de Lima and Laise de Holanda Cavalcanti

67'. Sporotheca with grayish or yellowish shades; capillitium decorated with warts or spines, not helicoidally arranged ........

68. Sporotheca subglobose to obpyriform, yellowish-gray or olivaceous; stalk filled at the base with subglobose or slightly angular cysts, 25-38 $\mu \mathrm{m}$; capillitium flexuous, greenish-yellow by transmitted light, with uniform filaments, 2-5 (-8) $\mu \mathrm{m}$ diam, decorated with warts and/or spinules, occasionally with clavate or rounded free ends

Arcyria pomiformis (Leers) Rostaf. ${ }^{(6)}$

68'. Sporotheca typically subcylindric, sometimes ovoid to subglobose, usually pale gray; stalk filled with globose cysts, 15-21 $\mu \mathrm{m}$ diam.; capillitium flexuous, hyaline by transmitted light, with filaments of lower portion irregular, smooth, 2-6 $(-10) \mu \mathrm{m}$ diam. and those of upper portion densely spinulose, more symmetrical, 1.5-4.0 $\mu \mathrm{m}$ diam

. Arcyria cinerea (Bull.) Pers. ${ }^{(1,3,4,5,6,8,8,10,11)}$

69. Sporophore plasmodiocarpic, linear, reticulate or ring-shaped; capillitium filaments with long spines of up to $5 \mu \mathrm{m}$ in length Perichaena chrysosperma (Curr.) Lister. ${ }^{(4,6,7,9)}$ 69'. Sporophore sporocarpic; capillitium filaments with constrictions, warts or short spines of up to $2 \mu \mathrm{m}$ in length ....... 70 70. Sporocarp stalked to almost sessile, isolated or rarely in groups of two or three; dehiscence irregular, areola-shaped or in

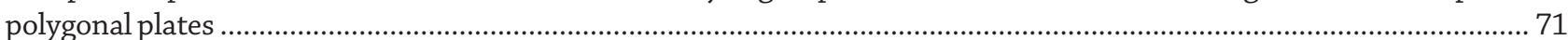
70'. Sporocarp sessile, densely grouped, seldom sparse, dehiscence circumcissile ...................................................... 72 71. Stalk developed, up to $0.7 \mathrm{~mm}$ long; dehiscence irregularly apical or areola-shaped; spores (8-) $9-10 \mu \mathrm{m}$....................... .. Perichaena pedata (Lister \& G. Lister) Lister ex E. Jahn. ${ }^{(1,2)}$ 71'. Stalk very short, up to $0.35 \mathrm{~mm}$ long or absent; dehiscence by polygonal plates, peridium remaining at base as a petaloid calyculus; spores 10-13.5 $\mu \mathrm{m}$ diam ................................................... Perichaena calongei Lado, D. Wrigley \& Estrada. ${ }^{(9)}$ 72. Sporotheca flattened, base fully supported by substrate, dehiscence line on bottom third, close to base; spores spinose, 9-11 $\mu$ m diam .................................................................................................................. Perichaena depressa Lib. ${ }^{(3,4,5,6,7,9)}$ 72'. Sporotheca subglobose, base not fully supported by substrate, average dehiscence line; spores faintly warted, 12-14 $\mu \mathrm{m}$ diam. Perichaena corticalis (Batsch) Rostaf. ${ }^{(3,4,6,7)}$

\section{Discusion}

The number of corticolous species of Myxomycetes currently known from CEPE corresponds to $23.5 \%$ of the total number recorded by Cavalcanti (2015) in northeastern Brazil for most ecological groups and vegetation types. Although this number is significant, half of the species were recorded from only one site within CEPE; eleven were collected in Seasonal Semideciduous Forests (Lowland) and 12 in Dense Ombrophilous Forest (Lowland) (Tab. 1). Comatricha longipila, Paradiachaeopsis longipes and Physarum decipiens were recorded as corticolous in CEPE only in Restinga of the state of Rio Grande do Norte. Although Comatricha pulchella, Physarum auriscalpium, Stemonaria fuscoides and $S$. irregularis have only been reported from mangroves of Rio Formoso (Tab. 1), they occur as lignicolous or suculenticulous at other localities in CEPE and in Caatinga and Cerrado vegetation in northeastern Brazil, with the exception of $S$. fuscoides (Rufino \& Cavalcanti 2007; Tenorio et al. 2009; Ferreira \& Cavalcanti 2011).

Among the 16 most common corticolous species at Great Mountains National Park (Snell \& Keller 2003), nine occur at CEPE, of which A. cinerea, Clastoderma debaryanum, $C$. confusa, $C$. violacea and E. minutum are the most common, being recorded in all vegetation types (Tab.1). Arcyria cinerea has been recorded in all regions of Brazil, occurring in Caatinga, Cerrado, Atlantic Forest, Mangrove and Restinga, where it occupies different micro-habitats, with decaying wood and bark of living trees being the most common (Cavalcanti 2015). Clastoderma debaryanum occurs worldwide and is considered among the most common species on living trees (Eliasson \& Keller 1996). In Brazil, this species has an extensive distribution, mainly in the Northeast Region (Bahia, Paraíba, Pernambuco, Piauí, Rio Grande do Norte), but there are also records in the Southeast (São Paulo) and South (Santa Catarina) regions, where they are corticolous and lignicolous in Atlantic Forest, Caatinga and Cerrado (Cavalcanti 2015). Cribraria confusa is one of the myxomycetes with the smallest sporocarps, being very hard to detect; this species is cited worldwide as corticolous on a variety of vegetation. In Brazil, this species has been recorded only in the Northeast Region (Alagoas, Maranhão, Pernambuco, Rio Grande do Norte, Sergipe), in Cerrado and moist forest environments (Cavalcanti 2015). Although rare, Cribraria confusa also is part of the CEPE lignicolous myxobiota, as reported by Cavalcanti et al. (2006) for Mata Bom Jesus of Usina Serra Grande in the municipality of São José da Laje, Alagoas. Cribraria violacea also produce small sporangia, and is very common on the cortex of living trees and, less frequently, on dead wood. In Brazil, this species has been recorded from almost all northeastern states, plus once in the South Region (Santa Catarina) and once in the Southeast Region (São Paulo), occurring as corticolous and lignicolous in the Atlantic Forest and Caatinga (Gusmão et al. 2005; Silva \& Cavalcanti 2010; Bezerra et al. 2011; Silva \& Cavalcanti 2012). Echinostelium minutum is known only from the Northeast (Alagoas, Bahia, Maranhão, Pernambuco) and 


\section{Corticolous myxobiota of the Pernambuco Center of Endemism, Brazil}

Table 1. Vegetation types where were found corticolous myxomycetes at the Pernambuco Center of Endemism (CEPE), Northeastern of Brazil. SDF = Seasonal Semideciduous Forest (Lowland), ODF= Dense Ombrophilous Forest (Lowland), MG= Mangrove and RT = Restinga.

\begin{tabular}{|c|c|c|c|c|c|}
\hline Species & SDF & ODF & MG & RT & $\begin{array}{c}\text { CEPE } \\
\text { Localities [\%] }\end{array}$ \\
\hline Arcyria cinerea & + & + & + & + & 75 \\
\hline Arcyria denudata & & + & & & 8 \\
\hline Arcyria ferruginea & & + & & & 8 \\
\hline Arcyria pomiformis & + & & & & 8 \\
\hline Badhamiopsis ainoae & + & & & & 8 \\
\hline Clastoderma debaryanum & + & + & + & + & 50 \\
\hline Collaria arcyrionema & & & + & + & 17 \\
\hline Comatricha elegans & & + & & + & 17 \\
\hline Comatricha laxa & + & + & & & 17 \\
\hline Comatricha longipila & & & & + & 8 \\
\hline Comatricha pulchella & & & + & & 8 \\
\hline Comatricha tenerrima & + & & & & 8 \\
\hline Craterium aureum & & + & & & 8 \\
\hline Craterium leucocephalum & & + & & & 8 \\
\hline Craterium paraguayense & & + & & & 8 \\
\hline Cribraria cancellata & + & + & & + & 25 \\
\hline Cribraria confusa & + & + & + & + & 58 \\
\hline Cribraria intricata & + & + & & + & 25 \\
\hline Cribraria microcarpa & + & + & & + & 58 \\
\hline Cribraria violacea & + & + & & + & 50 \\
\hline Diachea leucopodia & & & + & & 8 \\
\hline Dictydiaethalium plumbeum & + & & & & 8 \\
\hline Diderma chondrioderma & + & & & & 8 \\
\hline Didymium clavus & & & + & & 8 \\
\hline Didymium nigripes & + & & & & 8 \\
\hline Echinostelium minutum & + & + & & + & 58 \\
\hline Fuligo septica & & + & & & 8 \\
\hline Hemitrichia calyculata & + & & + & + & 33 \\
\hline Hemitrichia minor & & + & & & 8 \\
\hline Hemitrichia pardina & + & + & & & 17 \\
\hline Hemitrichia serpula & & + & & & 17 \\
\hline Licea biforis & & + & & & 8 \\
\hline Licea erecta & + & + & & & 17 \\
\hline Licea kleistobolus & & + & + & + & 25 \\
\hline Licea operculata & + & + & & & 25 \\
\hline Licea pedicellata & & + & & & 8 \\
\hline Lycogala exiguum & + & & & & 8 \\
\hline Macbrideola decapillata & + & & & + & 8 \\
\hline Macbrideola martinii & & + & & & 8 \\
\hline Macbrideola scintillans & & + & + & + & 25 \\
\hline Paradiacheopsis longipes & & & & + & 8 \\
\hline Paradiacheopsis rigida & + & + & & & 17 \\
\hline Perichaena calongei & & + & & & 8 \\
\hline Perichaena chrysosperma & + & + & & & 33 \\
\hline Perichaena corticalis & + & + & & & 33 \\
\hline Perichaena depressa & + & + & & & 50 \\
\hline Perichaena pedata & + & + & & & 17 \\
\hline Physarum album & & + & & & 8 \\
\hline Physarum auriscalpium & & & + & & 8 \\
\hline Physarum compressum & + & & & & 17 \\
\hline Physarum crateriforme & & + & & & 8 \\
\hline Physarum bogoriense & & + & & & 8 \\
\hline Physarum decipiens & & & & + & 8 \\
\hline
\end{tabular}


Table 1. Cont.

\begin{tabular}{|c|c|c|c|c|c|}
\hline Species & SDF & ODF & MG & RT & $\begin{array}{c}\text { CEPE } \\
\text { Localities }[\%]\end{array}$ \\
\hline Physarum echinosporum & & + & & + & 17 \\
\hline Physarum gyrosum & & + & & & 17 \\
\hline Physarum leucopus & & + & & & 8 \\
\hline Physarum leucopheum & & + & & & 8 \\
\hline Physarum melleum & + & & & & 8 \\
\hline Physarum nucleatum & + & & & & 17 \\
\hline Physarum oblatum & & + & & & 8 \\
\hline Physarum pezizoideum & & + & & & 8 \\
\hline Physarum roseum & + & & & + & 17 \\
\hline Physarum stellatum & + & + & & & 17 \\
\hline Physarum tenerum & & & + & & 8 \\
\hline Physarum viride & & + & & + & 17 \\
\hline Stemonaria fuscoides & & & + & & 8 \\
\hline Stemonaria longa & & + & & & 8 \\
\hline Stemonaria irregularis & & & + & & 8 \\
\hline Stemonitis axifera & + & + & & & 17 \\
\hline Stemonitis fusca & + & & + & + & 33 \\
\hline Stemonitis herbatica & & + & & & 8 \\
\hline Stemonitis splendens & & + & & & 8 \\
\hline Stemonitopsis typhina & & + & & & 8 \\
\hline Total & 33 & 48 & 15 & 21 & \\
\hline
\end{tabular}

Southeast (SP) regions of Brazil, occurring as corticolous in Caatinga, Cerrado, Atlantic Forest and Mangrove (Cavalcanti 2015); it is one of the first to sporulate, and its sporangium can be observed within two to three days after culture initiation.

Some corticolous species that occur in CEPE have been rarely recorded in Brazil. Among these are Perichaena calongei, Physarum gyrosum, Licea biforis and L. pedicellata, which have been recorded in Ombrophilous Forest of Biological Reserve in the municipality of Saltinho, and Comatricha longipila, Paradiachaeopsis longipes and Physarum decipiens, which were found in Restinga vegetation of Dunas do Natal State Park. Perichaena calongei was reported in Brazil for the first time by Araujo et al. (2015), who obtained it from moist chamber culture mounted with dead leaves and bark of living trees from Cerrado in the state of Goiás. This species was recorded for the first time in the Northeast Region in Ombrophilous Dense Submontane Forest of the Atlantic Forest in the state of Bahia, by moist-chamber culture mounted with lianas collected at Private Reserve of Natural Heritage (RPPN) Serra do Teimoso and, subsequently, at CEPE in Restinga of RPPN Mata Estrela in the state of Rio Grande do Norte (Cavalcanti et al. 2016).

Considering the myxobiota from REBIO Saltinho, one of the most humid environments among the 12 areas explored for myxomycetes in the CEPE, the present work documented 21 species (ca. of $44 \%$ of the species currently known from CEPE), occurring in 256 moist-chambers with bark of living trees. Although this is greater than the number of corticolous species observed by Stephenson et al. (2004) in the rainforest of the Maquipucuna Reserve in Ecuador (14 spp), it is lower than that encountered in temperate forests by Snell \& Keller (2003), who reported 84 species and 24 genera $(S / G=3.5)$ from 418 moist chamber cultures and Everhart \& Keller (2008), who recorded 46 species and 20 genera $(S / G=2.3)$ in 580 moist chambers.

The data presented herein are consistent with the observation that tropical corticolous myxobiota are less rich than those of temperate regions. However, the taxonomic diversity recorded at REBIO Saltinho $(S / G=2.3)$ and CEPE $(S / G=2.8)$, are equal to, or higher than, that of some temperate forests (e.g., Snell \& Keller 2003; Everhart \& Keller 2008).

\section{Acknowledgments}

We thank the UFPE Myxomycetes Laboratory members Andressa Vieira, Maria Chanti, Camila Vogeley, Antônia Aurelice A. Costa and Elaiza Rocha, for field and laboratory assistance. The Conselho Nacional de Desenvolvimento Científico e Tecnológico - CNPq (Proc. 305967/2009. 6 e.311340/2014-15) and the Fundação de Amparo à Ciência e Tecnologia de Pernambuco - FACEPE (Proc. BFP-00572.12/12), provided financial support.

\section{References}

Araujo JC, Lado C, Xavier-Santos S. 2015. Perichaena calongei (Trichiales): a new record of Myxomycetes from Brazil. Current Research in Environmental \& Applied Mycology 5: 352-356. 
Basanta DW. 1998. Myxomycetes from the bark of the evergreen oak Quercus ilex. Anales del Jardín Botanico de Madrid 56: 1-14

Bezerra ACC, Cavalcanti LH. 2007. Mixobiota corticícola de Terminalia catappa L. (Combretaceae). Sitientibus, Serie Ciências Biológicas 7: 154-160.

Bezerra ACC, Costa AAA, Cavalcanti LH. 2011. Myxomycetes occurring on Cecropia adenopus (Cecropiaceae) in fragments of the Atlantic Rainforest. Acta Botanica Brasílica 25: 11-16.

Bezerra ACC, Nunes AT, Costa AAA, Ferreira IN, Bezerra MFA, Cavalcanti LH. 2007. Mixobiota do Parque Estadual das Dunas de Natal. Revista Brasileira de Biociências 5: 30-32.

Cavalcanti LH. 1974. Mixomicetos corticícolas do Cerrado de Emas (Pirassununga - São Paulo). MSc Thesis, Universidade de São Paulo, Brazil.

Cavalcanti LH. 2015. Myxomycetes. In: Lista de Espécies da Flora do Brasil. Jardim Botânico do Rio de Janeiro. http://floradobrasil.jbrj. gov.br. 9 Dez. 2015.

Cavalcanti LH, Bezerra ACC, Barbosa DI, et al. 2016. Occurrence and distribution of Perichaena (Trichiaceae, Myxomycetes) in the Brazilian Northeastern region. Acta Botanica Brasilica 30: 102-111.

Cavalcanti LH, Damasceno G, Bezerra ACC, Costa AAA. 2014. Mangrove myxomycetes: species occurring on Conocarpus erectus L. (Combretaceae). Sydowia 66: 183-190.

Cavalcanti LH, Tavares HFM, Nunes ATF, Silva CF. 2006. Mixomicetos. In: Pôrto CK, Almeida-Cortez JC, Tabarelli M. (eds.) Diversidade Biológica e Conservação da Floresta Atlântica ao Norte do Rio São Francisco. Brasília, Ministério do Meio Ambiente (Série Biodiversidade 14). p. 53-72.

Coimbra-Filho AF, Câmara IG. 1996. Os limites originais do bioma Mata Atlântica na Região Nordeste do Brasil. Rio de Janeiro, Fundação Brasileira para a Conservação da Natureza (FBCN).

Damasceno G, Tenorio JCG, Cavalcanti LH. 2011. Stemonitaceae (Myxomycetes) in Brazilian mangroves. Sydowia 63: 9-22.

Eliasson UH, Keller HW. 1996. The genus Clastoderma: taxonomic evaluation and infraspecific variation of $C$. debaryanum. Mycological Research 100: 601-609.

Everhart SE, Keller HW. 2008. Life history strategies of corticolous myxomycetes: the life cycle, plasmodial types, fruiting bodies, and taxonomic orders. Fungal Diversity 29: 1-16.

Farr ML. 1976. Myxomycetes Flora Neotropica. Monograph 16. New York, New York Botanical Garden.

Ferreira IN, Cavalcanti LH. 2011. Myxomycetes associados a cactáceas no agreste e sertão de Pernambuco, Brasil. Biotemas 24:1-11.

Gray WD, Alexopoulos CJ. 1968. Biology of the Myxomycetes. New York, Ronald Press Company.

Gusmão LFP, Góes-Neto A, Cruz ACR. 2005. Fungos. In: Juncá FA, Funch L, Rocha W. (orgs.) Biodiversidade e Conservação da Chapada Diamantina. Brasília, Ministério do Meio Ambiente. p. 225-242.

Ing B. 1994. The phytosociology of myxomycetes. New Phytologist 126: 175-201.

Keller HW, Everhart SE. 2010. Importance of Myxomycetes in Biological Research and Teaching Winter. Fungi 3: 13-27.

Lado C, Pando F. 1997. Flora Micológica Ibérica. Myxomycetes I.V.2. Berlin, Cramer.

Lado C. 2015. An on-line nomenclatural information system of Eumycetozoa. http://www.nomen.eumycetozoa.com. 9 Dez. 2015.

Madelin MF. 1984. Myxomycete data of ecological significance. Mycological Society 83: 1-19.

Martin GW, Alexopoulos CJ. 1969. The Myxomycetes. Iowa, University of Iowa Press.

Mitchell DW. 2004. A key to the corticolous myxomycetes. Systematics and Geography of plants 74: 261-285.

Novozhilov YK, Mitchell DW, Schnittler M. 2003. Myxomycete biodiversity of the Colorado Plateau. Mycological Progress 2: 243-258.
Novozhilov YK, Schnittler M, Stephenson SL. 1999. Myxomycetes of the Taimyr Peninsula (north-central Siberia). Karstenia 39: 77-97.

Novozhilov YK, Zemlianskaia I, Schnittler M, Stephenson S. 2006. Myxomycete diversity and ecology in the arid regions of the Lower Volga River Basin (Russia). Fungal Diversity 23: 193-241.

Poulain M, Meyer M, Bozonnet J. 2011. Les Myxomycètes. Sévrier, Fédération Mycologique et Botanique Dauphiné-Savoie.

Roda AS, Pereira GA. 2006. Distribuição recente e conservação das aves de rapina florestais do Centro de Endemismo Pernambuco. Revista Brasileira de Ornitologia 14: 331-344.

Rolim GS, Camargo MBP, Lania DG, Moraes JFL. 2007. Classificação climática de Köppen e de Thornthwaite e sua aplicabilidade na determinação de zonas agroclimáticas para o estado de São Paulo. Bragantia 66: 711-720.

Rufino MUL, Cavalcanti LH. 2007. Alterations in thelignicolousmyxomycete biota over two decades at the Dois Irmãos Ecologic State Reserve, Recife, Pernambuco, Brazil. Fungal Diversity 24: 159-171.

Schnittler M. 2001. Ecology of myxomycetes of a winter-cold desert in western Kazakhstan. Mycologia 93: 653-669.

Silva CF, Cavalcanti LH. 2010. Myxobiota of the Brazilian Atlantic Forest: Species on oil palm tree (Elaeis guineensis Jacq., Arecaceae). Rodriguésia 61: 575-583.

Silva NA, Cavalcanti LH. 2012. Myxomycetes ocorrentes em áreas de caatinga e brejo de altitude no sertão de Pernambuco, Brasil. Acta Botanica Brasilica 26: 901-915.

Silva VPR, Pereira ERR, Azevedo PV, Sousa FAZ, Sousa IF. 2011. Análise da pluviometria e dias chuvosos na região Nordeste do Brasil. Revista Brasileira de Engenharia Agrícola e Ambiental 15: 131-138.

Snell L, Keller HW. 2003. Vertical distribution and assemblages of corticolous myxomycetes on five tree species in the Great Smoky Mountains National Park. Mycologia 95: 565-576.

Stephenson SL, Feest A. 2012. Ecology of soil eumycetozoans. Acta Protozoologica 51: 201-208.

Stephenson SL, Kalyanasundaram I, Lakhanpal TN. 1993. A comparative biogeographical study of myxomycetes in the mid-Appalachians of eastern North America and two regions of India. Journal of Biogeography 20: 645-557.

Stephenson SL, Novozhilov YK, Schnittler M. 2000. Distribution and ecology of myxomycetes in high-latitude regions of the northern hemisphere. Journal of Biogeography 27: 741-754.

Stephenson SL, Schnittler M, Lado C. 2004. Ecological characterization of a tropical Myxomycete assemblage - Maquipucuna Cloud Forest Reserve, Ecuador. Mycologia 96: 488-497.

Stephenson SL, Schnittler M, Novozhilov YK. 2008. Myxomycete diversity and distribution from the fossil record to the present. Biodiversity and Conservation 17: 285-301.

Tabarelli M, Santos AMM. 2004. Uma breve descrição sobre a história natural dos brejos nordestinos. In: Pôrto KC, Cabral JJP, Tabarelli M. (eds.) Brejos de altitude em Pernambuco e Paraíba: história natural, ecologia e conservação. Brasília, Ministério do Meio Ambiente. p. 17-24.

Tabarelli M, Siqueira Filho JA, Santos AMM. 2006. A Floresta Atlântica ao Norte do Rio São Francisco. In: Pôrto CK. (ed.) Diversidade biológica e conservação da Floresta Atlântica ao Norte do Rio São Francisco. Brasília, Ministério do Meio Ambiente. p. 25-40.

Tenório JG, Bezerra MFA, Costa AAA, Cavalcanti LH. 2009. Mixobiota do Parque Nacional Serra de Itabaiana, SE, Brasil: Stemonitales. Acta Botanica Brasilica 23: 644-656.

Tran HTM, Stephenson SL, Hyde KD, Mongkolporn O. 2006. Distribution and occurrence of myxomycetes in tropical forests of northern Thailand. Fungal Diversity 22: 227-242.

Uchoa Neto CAM, Tabarelli M. 2002. Diagnóstico e estratégia de conservação do Centro de Endemismo Pernambuco. Recife, Conservation International do Brasil. 